\title{
Characterisation of Partial Discharge Behaviour in Liquid Nitrogen
}

\author{
D J Swaffield, P L Lewin, Y Tian, G Chen and S G Swingler* \\ University of Southampton, Electronics and Computer Science, Highfield, Southampton, SO17 1BJ, UK \\ *formerly, National Grid Transco plc, Warwick, UK \\ djs@ecs.soton.ac.uk
}

\begin{abstract}
Interest in superconductors for use in the transmission and distribution of power has grown since the advent of High Temperature Superconductors (HTS). The benefits of reduced ohmic losses leading to greater operational efficiency appear attractive to power transmission and distribution companies. Potentially this technology will increase power transfer for the same or lower voltage and for the same or smaller physical dimensions. For the successful employment of this technology in the field, power utilities must be convinced of the operational reliability of designs. Many of these designs make use of liquid nitrogen as the system coolant and also as a dielectric fluid. It is therefore vital that liquid nitrogen be well characterised as a dielectric medium. This paper describes an experiment to characterise partial discharge behaviour in a non-uniform field geometry. A technique is described which allows synchronised capture and measurement of apparent charge and images of density change streamer activity. $\varphi \mathrm{q} \mathbf{n}$ analysis of ac results has been completed and characterisation of partial discharge behaviour is presented and discussed.
\end{abstract}

\section{INTRODUCTION}

Within existing prototype designs for High Temperature Superconducting (HTS) equipment, liquid nitrogen is employed in the dual role as coolant and as a dielectric. Liquid nitrogen has a high dielectric strength, comparable to dielectric liquids at room temperature [1]. Liquid nitrogen breakdown data displays volume and area effects and are dependant on other parameters including purity, electrode material and surface condition [2]. Due to a narrow liquid temperature range and low latent heat of vaporization the cryogenic liquid insulation suffers from the increased potential of density change to vapour. The increase in vapour in the insulation gap has been seen to reduce the breakdown voltage. Vapour can be introduced into the bulk liquid by nucleate boiling from conductors undergoing quench, due for example, to constraints in the design of an ambient to cryogenic current lead-through. Alternatively vapour may be introduced from electrical discharges into the liquid as shown in this experiment.

A non-uniform point-plane field geometry has been used to allow study of the physics of the breakdown mechanism. To study streamer growth without causing a total discharge two routes have been pursued in the literature. Firstly a transient voltage impulse can be applied to the sample, several works have been published taking this approach [3-5]. For study of partial breakdown under ac voltage a composite system is preferred. A stable partial discharge is difficult to achieve without a solid insulator to prevent total discharge because in a liquid the voltage for discharge inception is higher than that for sustained discharge, i.e. the initial discharge leads to total failure between electrodes for small gaps. Partial discharge (pd) under ac conditions has been examined in [6] for a triple junction, and [7] for point plane. Point-plane samples are used extensively to produce a strongly divergent field. In this study partial discharge is characterised for $50 \mathrm{~Hz}$ ac voltage applied to a point-plane geometry. $\varphi \mathrm{q} \mathrm{n}$ analysis of ac results has been completed and characterisation of partial discharge behaviour is presented and discussed.

\section{EXPERIMENTAL SETUP}

The experimental setup is shown in Figure 1. The sample consists of a tungsten needle with a radius of $2.7 \pm 0.4 \mu \mathrm{m}$ a $20^{\circ}$ apex angle and a shank diameter of $1 \mathrm{~mm}$. This is earthed and positioned below a plane electrode covered with a $4 \mathrm{~mm}$ thick plate of Glass Reinforced Resin (GRR), Tufnol ${ }^{\circledR}$ Grade10G/40. The liquid nitrogen filled gap between needle tip and GRR was $2.6 \mathrm{~mm}$. The test sample is enclosed within a purpose built cryostat that allows application of voltage, pd free to $40 \mathrm{kV}$ and controlled temperatures down to $63.05 \mathrm{~K}$ [8]. Following vacuuming of the cryostat inner vessel to remove moisture, it was filled with commercial grade liquid nitrogen.

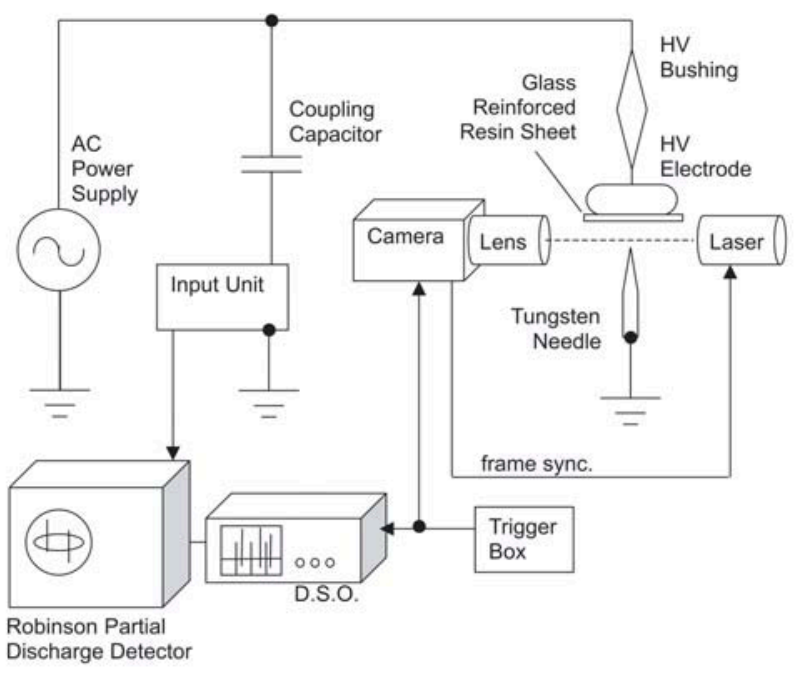

Figure 1 - Schematic diagram of experimental arrangement. 


\section{Experimental Procedure}

AC voltage was applied to the plane electrode at stages between 18.3 to $26.4 \mathrm{kV}$. The camera was triggered simultaneously with the digital storage oscilloscope (DSO) connected to capture PD activity measured via a Robinson PD detector Type 700. The DSO captured PD data for 200 cycles of applied ac voltage at $50 \mathrm{~Hz}$ power frequency.

Partial discharge signals have been correlated to images of density change streamers resulting from discharges, Figures 2 and 3. These were captured at 5000 frames per second (fps) using a high frequency pulse laser with a pulse duration of 30ns to stroboscopically backlight the needle sample. A microscope lens with long working distance was used to magnify the density charge streamer images. Images captured are 512 by 512 pixels, which with a pixel viewing an area of $2.42 \mu \mathrm{m}$ square equates to a full frame size for Figures 2 and 3 of $1.24 \mathrm{~mm}$ square.

Since it is not possible to measure the true charge at a discharge site the electrical circuit was calibrated prior to use. This was performed by employing a standard technique; injecting pulses of known charge and measuring the output signal seen on the pd detector and recorded on the dso. This allows a measure of "apparent charge" for each density charge streamer recorded.

Calculating the electric stress at the needle tip is difficult for several reasons. A typical approach is to use a hyperboloid to plane approximation to solve an expression for electric stress. This assumes that the needle geometry is regular and is close to a hyperboloid in geometry. Second, such an expression assumes no space charge effects. This is unlikely for this system as shown by the advancing phase shift with increasing voltage; seen later in Figures 4 and 5. The discharge current leads the voltage indicating the storage of charge. Such expressions are commonly used to provide an approximation for point plane systems, but this is further complicated in this case due to the inclusion of the GRR sheet with its own space and surface charge effects.

\section{Analysis, $\varphi$ q n plotting}

The partial discharge data captured at two voltage levels and two temperatures have been analysed using $\varphi \mathrm{q} \mathrm{n}$ calculation, Figures 4, 5 and 6. Partial discharges are recorded as a phase location and maximum amplitude. The $50 \mathrm{~Hz}$ power cycle, period $20 \mathrm{~ms}$, is divided into 200 phase windows and 100 charge amplitude divisions. Cumulative numbers of discharges falling into these windows are calculated and plotted to provide a diagrammatical representation of partial discharge and allows comparison of activity under different test conditions. Since the zero crossing detection circuit has a delay that may be up to $11 \mu \mathrm{s}$ and a single discharge pulse may have a pulse width of up to $40 \mu$ s at the dso. The combined error may therefore be as much as $51 \mu \mathrm{s}$, equating to a 0.918 degrees phase shift. However this is less than one phase window width of 1.8 degrees. Therefore, over the whole cycle errors will not significantly influence the shape of the $\varphi \mathrm{q} n$ plots.

\section{RESULTS AND DISCUSSION}

Two types of density change streamers have been observed, following different classification to authors studying impulse voltages these can be considered as "filamentary" and "bush like". Filamentary streamers appear in shape as single or multi-strand discharges with well defined branches along the discharge path from the needle tip toward the plane; these are only seen in the negative half-cycle i.e. when the needle tip is at higher potential than the plane electrode. Figure 2 shows a typical filamentary streamer.

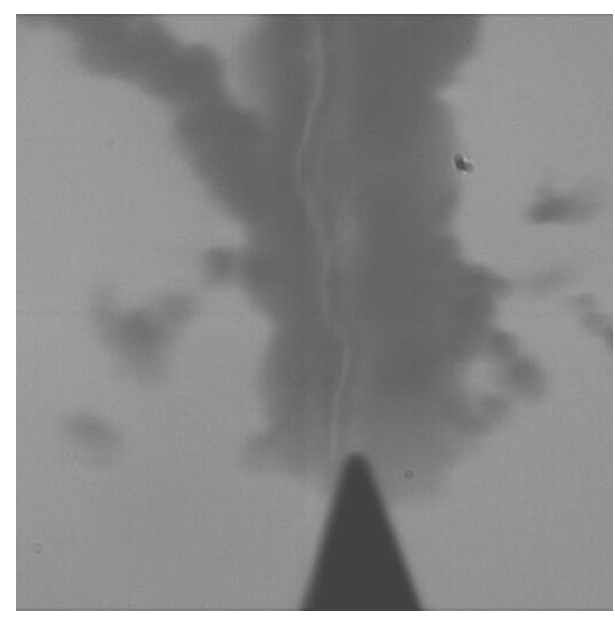

Figure 2 - Typical positive filamentary discharge with visible streamer, apparent charge of $369 \mathrm{pC}$ recorded at $266.796 \pm 0.198$ degrees phase angle.

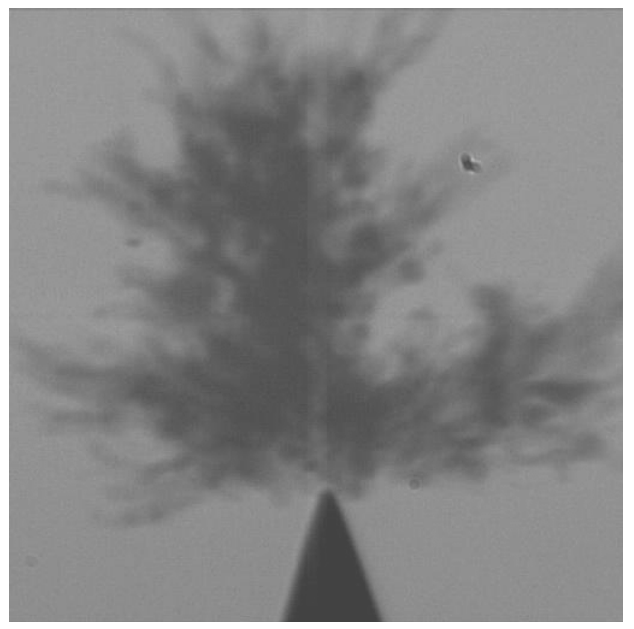

Figure 3 - Typical negative bush like discharge, with apparent charge of $170 \mathrm{pC}$ recorded at $80.190 \pm 0.198$ degrees phase angle. 
Figure 3 shows a typical bush like streamer discharge; these are characterised visually as having many branches spread out in all directions appearing as a rough edge to the density change streamer. These are typical of the positive half cycles, i.e. when the needle tip is at a lower potential to the plane electrode. Bush like streamers have been observed in the negative half-cycle, but filamentary streamers such as Figure 2 have never been seen in the positive half cycle.

With the needle at lower potential than the plane, after an initial bush discharge the vapour formed from a discharge is quickly swept up toward the plane. Discharges in this halfcycle are repetitive and frequent, the next discharge will initiate before the vapour from the previous discharge has fully cleared the camera shot. This is in contrast with the positive needle discharge. In this case vapour is formed as rapidly, i.e. less than one frame $(200 \mu \mathrm{s})$, but the vapour remains in the camera shot up to five times longer. This is enough time for bubbles to form and more slowly drift up, or liquefy. These observations may suggest that the vapour after a negative needle discharge is charged and is swept away due to electrophoresis. Whereas vapour bubbles formed from the positive needle discharge may be only weakly charged and drift upward under buoyancy force alone.

The resultant $\varphi \mathrm{q} \mathrm{n}$ plots show the change in character of the discharges as the applied $50 \mathrm{~Hz}$ voltage is increased from $18.3 \mathrm{kV}$ to $26.4 \mathrm{kV}$, Figures 4 and 5 respectively. For images captured at $26.4 \mathrm{kV}$ at $75.7 \pm 0.2 \mathrm{~K}$, corresponding to Figure 5, fluid currents presumed due to electroconvection are visible, unlike images corresponding to Figure 4, taken at $18.3 \mathrm{kV}$ at $76.0 \pm 0.3 \mathrm{~K}$. The results show that there are common features to partial discharge activity for all the temperatures and applied voltages investigated. The maximal partial discharges seen in the positive and negative half-cycles are approximately equal. As has been described more activity is seen in the positive half-cycle when the needle is at a lower potential to the plane electrode. This again demonstrates a difference in mechanism of discharge. Here electrons are readily supplied from the high electric stress at the needle tip; these are attracted to the plane electrode. Scattering of electron paths occurs because of the repelling like charge leading to the bush like structure observed in Figure 3. This also accounts for the observed spreading of the base of the bush structure away from the needle tip as the repetitive discharges continue and the initial electric field profile is distorted by the movement of charge.

The average discharge magnitude is higher in the negative half-cycle when the needle tip is at a higher potential than the plane electrode. Electron scavenging must occur from the liquid nitrogen and free electrons in the liquid are not so readily available. With increasing potential eventually there is a breakdown to the needle tip with the electrons directed toward the needle tip leading to a narrow conduction path, as seen in the example Figure 2.

As the applied voltage is increased from $18.3 \mathrm{kV}$ to $26.4 \mathrm{kV}$ the discharge activity increases, the maximum cumulative number of discharges in the positive half cycle rises by $31 \%$ and there is a phase advance of approximately 20 degrees for the discharge activity peaks in both half-cycles. In addition the average charge per phase window increases for the positive half-cycle and the activity in both half-cycles spreads over a larger phase range.

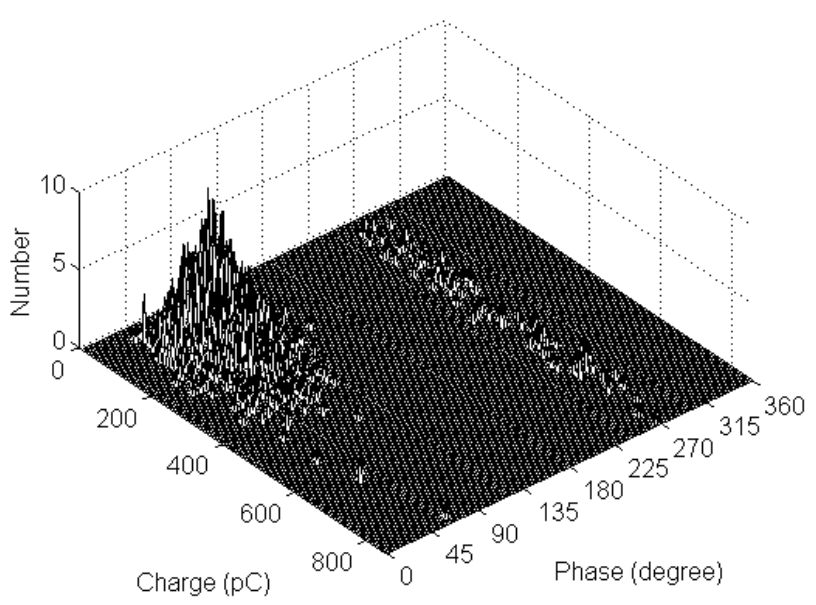

Figure 4 - pqn plot with an applied voltage of $18.3 \mathrm{kV}$ at $76.0 \pm 0.3 \mathrm{~K}$

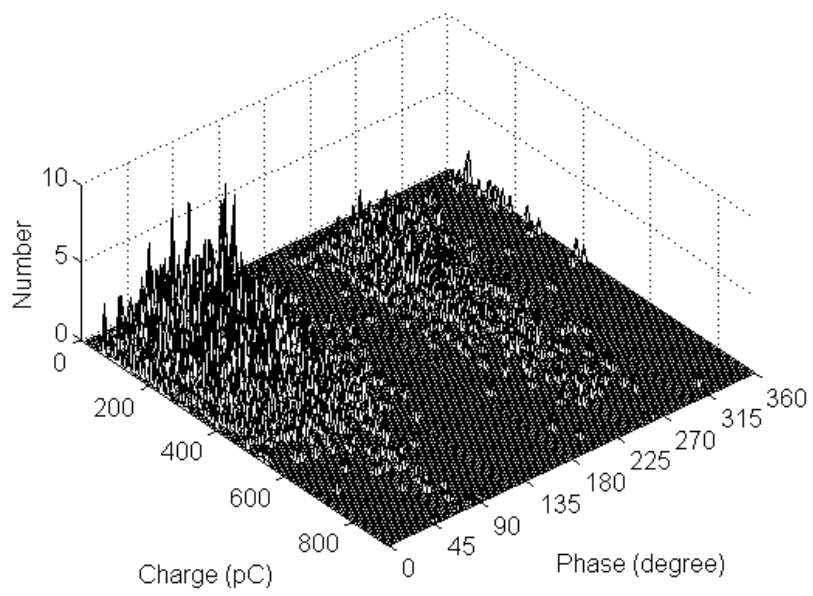

Figure 5 - $\varphi q n$ plot with an applied voltage of $26.4 \mathrm{kV}$ at $75.7 \pm 0.2 \mathrm{~K}$

A comparison of the $\varphi \mathrm{q} \mathrm{n}$ plots in Figures 5 and 6 shows the change in character of the discharges as the temperature of the liquid nitrogen/GRR composite insulation is reduced from $75.7 \mathrm{~K}$ to $63.7 \mathrm{~K}$ respectively. As with the conditions corresponding to Figure 5; fluid currents presumed due to electroconvection are seen in images captured for the conditions; $26.5 \mathrm{kV}$ at $63.7 \pm 0.1 \mathrm{~K}$, corresponding to Figure 6 . Reducing the temperature to $63.7 \mathrm{~K}$ narrows the phase range 
over which activity is seen for both halves of the cycle and the peaks of the cumulative numbers retard back toward the applied voltage peaks.

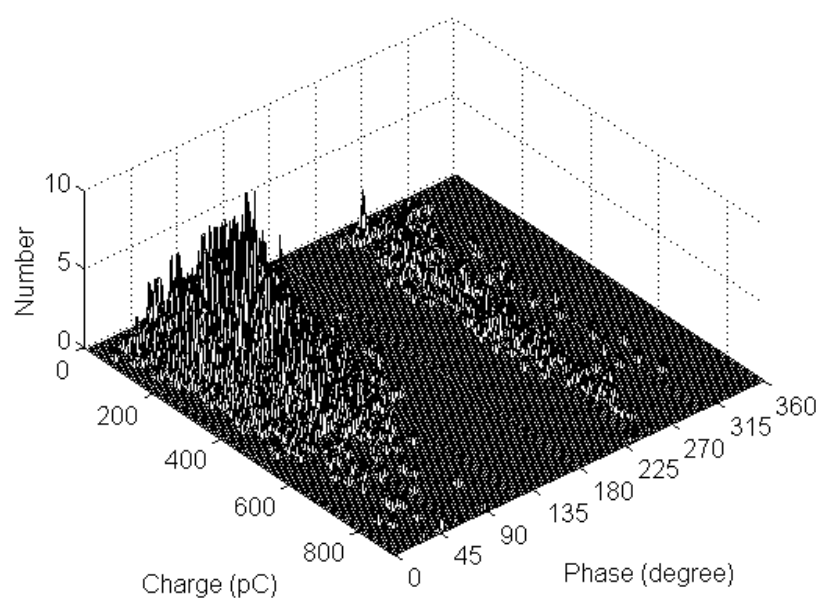

Figure 6 - $\varphi q n$ plot with an applied voltage of $26.5 \mathrm{kV}$ at $63.7 \pm 0.1 \mathrm{~K}$.

After the experimental run was complete, the needle and GRR were removed for inspection. This showed that the needle tip had been eroded by the discharges. The surface of the needle was examined under a microscope and observed to be irregular in shape and pitted; a measurement of the radius was therefore approximated to be $15.1 \pm 0.4 \mu \mathrm{m}$. The GRR sheet surface had also been damaged. A crater was formed directly above the position of the needle tip extending through several layers of the woven glass filler.

\section{CONCLUSIONS}

A method for capture of time correlated sequences of electrical discharge signals and images have been developed. This has been used to investigate the partial breakdown of liquid nitrogen in a point plane system of liquid nitrogen and GRR sheet. Using this method density change streamers can be imaged. Density change streamers are formed by heating the liquid nitrogen to a level above its vaporization point. Capture of long sequences of the resulting vapour generation in the insulation gap is possible by this method.

It has been seen that there are two mechanisms for partial breakdown, one leading to bush like streamers. The other, leads to filamentary streamers, which are only found when the needle tip is at a higher potential than the plane electrode. It is observed that dielectrically weaker nitrogen gas may be left in the liquid insulation gap after a partial discharge event and long enough to initiate further discharge activity.

$\varphi \mathrm{q} n$ plots have been computed to allow characterisation of behaviour for increasing voltage and decreasing temperature. These show an advance of discharge activity as the voltage is increased. This advance in discharge current is indicative of an increase in charge storage or capacitance in the system. This is expected to be a result of the GRR sheet surface and space charge effects becoming more prominent as the applied voltage is increased. The effect of a decrease in temperature is the retard the discharge activity reducing the capacitive effect of the GRR sheet. The sub-cooling of the nitrogen also suppresses discharge activity causing the narrowing of the phase range over which activity can occur.

Both the needle and the GRR sheet have been damaged by the experiments. This investigation has examined the character of partial discharge activity and provides evidence of possible failure mechanisms of interest to the design of composite cryogenic insulation systems.

\section{ACKNOWLEDGMENTS}

David J Swaffield is funded under EPSRC Grant GR/R/02610. The financial support of National Grid Transco for this project is gratefully acknowledged.

\section{REFERENCES}

1. Gerhold, J. "Dielctric breakdown of cryogenic gases and liquids", Cryogenics. 1979, pp. 571-584.

2. Hayakawa, N., Sakakibara, H., Goshima, H., Hikita, M., Okubo, H. "Breakdown mechanism of liquid nitrogen viewed from area and volume effects", IEEE Transactions on Dielectrics and Electrical Insulation. Vol. 4 No. 1, 1997, pp. 127-134.

3. Hanaoka, R., Ishibashi, R., Kasama, M., Uchiyama, A., Kawaguchi, A. "Pre-breakdown current in liquid nitrogen under dc nonuniform field", Nuclear Instruments and Methods in Physics Research. Vol. A327, 1993, pp. 107-110.

4. Yamazawa, K., Yamashita, H. "Prebreakdown Density Change Streamer in Liquid Nitrogen", Japanese Journal of Applied Physics. Vol. 36 Part 1 No. 10, 1997, pp. 6437-6443. 5. Frayssines, P. E., Lesaint, O., Bonifaci, N., Denat, A., Lelaidier, S., Devaux, F. "Prebreakdown phenomena at high voltage in liquid nitrogen and comparison with mineral oil", IEEE Transactions on Dielectrics and Electrical Insulation. Vol. 9 No. 6, 2002, pp. 899-909.

6. Suehiro, J., Matsumoto, Y., Imasaka, K., Hara, M. "Partial discharge induced bubbles generated in subcooled liquid nitrogen at atmospheric pressure". 13th International Symposium on High Voltage Engineering (ISH), 2003. pp. 153.

7. Takahasi, Y., Ohtsuka, K. "Corona discharges and bubbling in liquid nitrogen", Journal of Physics D (Applied Physics). Vol. 8, 1974, pp. 165-169.

8. Swaffield, D. J., Lewin, P. L., Chen, G., Swingler, S. G. "The influence of bubble dynamics in liquid nitrogen with applied electric fields on superconducting power apparatus". The 13th International Symposium on High Voltage Engineering, 2003. pp. 457. 